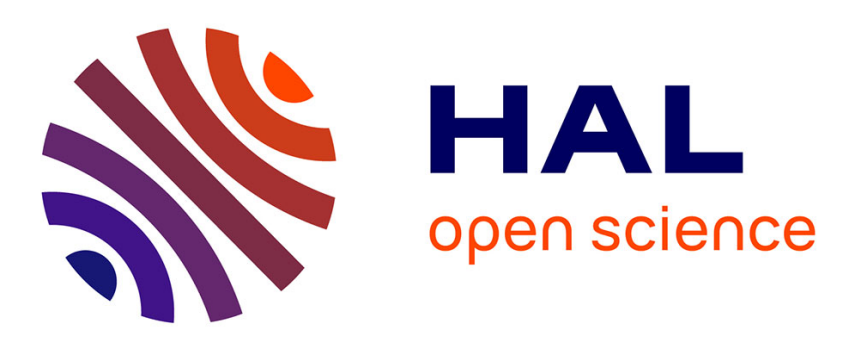

\title{
An illumination problem: optimal apex and optimal orientation for a cone of light.
}

\author{
Annabella Astorino, Manlio Gaudioso, Alberto Seeger
}

\section{To cite this version:}

Annabella Astorino, Manlio Gaudioso, Alberto Seeger. An illumination problem: optimal apex and optimal orientation for a cone of light.. Journal of Global Optimization, 2014, 11 (1), pp.128-149. 10.1007/s10898-013-0071-0 . hal-01326089

\section{HAL Id: hal-01326089 \\ https://hal.science/hal-01326089}

Submitted on 8 Nov 2017

HAL is a multi-disciplinary open access archive for the deposit and dissemination of scientific research documents, whether they are published or not. The documents may come from teaching and research institutions in France or abroad, or from public or private research centers.
L'archive ouverte pluridisciplinaire HAL, est destinée au dépôt et à la diffusion de documents scientifiques de niveau recherche, publiés ou non, émanant des établissements d'enseignement et de recherche français ou étrangers, des laboratoires publics ou privés. 


\title{
An illumination problem: optimal apex and optimal orientation for a cone of light
}

\author{
Annabella Astorino - Manlio Gaudioso • \\ Alberto Seeger
}

\begin{abstract}
Let $\left\{a_{i}: i \in I\right\}$ be a finite set in $\mathbb{R}^{n}$. The illumination problem addressed in this work is about selecting an apex $z$ in a prescribed set $Z \subseteq \mathbb{R}^{n}$ and a unit vector $y \in \mathbb{R}^{n}$ so that the conic light beam

$$
C(z, y, s):=\left\{x \in \mathbb{R}^{n}: s\|x-z\|-\langle y, x-z\rangle \leq 0\right\}
$$

captures every $a_{i}$ and, at the same time, it has a sharpness coefficient $s \in[0,1]$ as large as possible.
\end{abstract}

Keywords Illumination problem $\cdot$ Revolution cone $\cdot$ Aperture angle $\cdot$ Sharpness coefficient $\cdot$ Max-min problem

\section{Introduction}

There is a broad literature devoted to various sorts of illumination and/or visibility problems; see for instance $[1,7,9,22]$. Most illumination problems arising in practice are stated in dimensions two and three, but one could equally well consider higher dimensions. Let $\mathbb{R}^{n}$ be equipped with its usual inner product $\langle\cdot, \cdot\rangle$ and the associated norm $\|\cdot\|$. Mathematically speaking, a conic light beam is a generalized revolution cone

\footnotetext{
A. Astorino

Istituto di Calcolo e Reti ad Alte Prestazioni,

Consiglio Nazionale delle Ricerche (CNR), 87036 Rende, Italy

e-mail: astorino@icar.cnr.it

M. Gaudioso $(\varangle)$

Dipartimento di Ingegneria Informatica, Modellistica, Elettronica e Sistemistica, Università delle Calabria, 87036 Rende, Italy

e-mail: gaudioso@deis.unical.it

A. Seeger

Department of Mathematics, University of Avignon, 33 rue Louis Pasteur, 84000 Avignon, France

e-mail: alberto.seeger@univ-avignon.fr
} 


$$
C(z, y, s):=\left\{x \in \mathbb{R}^{n}: s\|x-z\|-\langle y, x-z\rangle \leq 0\right\}
$$

depending on three parameters:

- The apex $z$ of the cone is the geometric locus at which a source of light is placed. Throughout this work, $z$ is selected from a closed set $Z \subseteq \mathbb{R}^{n}$. One refers to (1) as a "generalized" revolution cone because the apex $z$ is not necessarily the origin $\mathbf{0}$ of the space $\mathbb{R}^{n}$.

- The axis $y \in \mathbb{R}^{n}$ is a nonzero vector that determines the orientation of the light beam. The axis can always be normalized, so one takes it from the unit sphere $\mathbb{S}_{n}:=\left\{y \in \mathbb{R}^{n}\right.$ : $\|y\|=1\}$.

- The scalar $s \in[0,1]$ is the sharpness coefficient of the light beam. One refers to $\vartheta:=2 \arccos s$ as the aperture angle of (1). So, maximizing the sharpness coefficient amounts to minimizing the aperture angle.

One may think of (1) as the visible or illuminated region of the space $\mathbb{R}^{n}$. Thus, a sample $A=\left\{a_{i}: i \in I\right\}$ is illuminated by the conic light beam (1) if and only if $A \subseteq C(z, y, s)$. The smallest aperture angle illumination problem reads then as follows:

$$
(\mathcal{P})\left\{\begin{array}{l}
\text { maximize } s \\
(z, y, s) \in Z \times \mathbb{S}_{n} \times[0,1] \\
f_{i}(z, y, s) \leq 0 \text { for all } i \in I,
\end{array}\right.
$$

where each $f_{i}: \mathbb{R}^{2 n+1} \rightarrow \mathbb{R}$ is defined by

$$
f_{i}(z, y, s):=s\left\|a_{i}-z\right\|-\left\langle y, a_{i}-z\right\rangle .
$$

The above maximization problem will be studied in detail in Sects. 2 and 3, both theoretically and algorithmically. We shall be concerned also with a sort of dual version of (2). Up to a closure operation, the invisible or penumbra region

$$
D(z, y, s):=\left\{x \in \mathbb{R}^{n}: s\|x-z\|-\langle y, x-z\rangle \geq 0\right\}
$$

corresponds to the set-complement of (1). The formulation of the largest aperture angle penumbra problem is as follows:

$$
\text { (Q) }\left\{\begin{array}{l}
\text { minimize } s \\
(z, y, s) \in Z \times \mathbb{S}_{n} \times[0,1] \\
f_{i}(z, y, s) \geq 0 \text { for all } i \in I .
\end{array}\right.
$$

Despite their striking similarity, the problems (2) and (4) are structurally different. Note for instance that the illuminated region (1) is convex, while the penumbra region (3) is nonconvex. Section 4 will be devoted to the analysis of the penumbra problem. One should not view (2) and (4) as a pair of dual problems in the sense of convex optimization theory. The word duality is used here in a loose sense.

\section{Theoretical aspects of the illumination problem}

\subsection{Preliminary material on supporting cones}

The symbols $P^{c}$, int $(P)$, and $\operatorname{bd}(P)$ denote respectively the set-complement, the interior, and the boundary of the polytope $P:=\operatorname{co}\left\{a_{i}: i \in I\right\}$. We use also the notation

$$
\Gamma(y, s):=C(\mathbf{0}, y, s) \text { and } T(z):=\mathbb{R}_{+}(P-z) .
$$


Both sets in (5) are closed convex cones with apices at $\mathbf{0}$. The first one is a standard revolution cone, whereas the second one is a polyhedral (or finitely generated) convex cone:

$$
T(z)=\left\{\sum_{i \in I} \mu_{i}\left(a_{i}-z\right): \mu_{i} \geq 0 \text { for all } i \in I\right\} .
$$

One refers to $T(z)$ as the supporting cone to $P$ at $z$. Such cone is of special interest when $z$ belongs to $P^{c}$.

The continuity result stated in Proposition 2.1 is recalled here for the sake of convenience. One considers the set $\Xi\left(\mathbb{R}^{n}\right)$ of nontrivial closed convex cones in $\mathbb{R}^{n}$ equipped with the spherically truncated Pompeiu-Hausdorff metric

$$
\varrho\left(K_{1}, K_{2}\right):=\operatorname{haus}\left(K_{1} \cap \mathbb{S}_{n}, K_{2} \cap \mathbb{S}_{n}\right),
$$

where haus $(\cdot, \cdot)$ refers to the classical Pompeiu-Hausdorff metric on the collection of nonempty compact sets, i.e.,

$$
\operatorname{haus}\left(\Omega_{1}, \Omega_{2}\right):=\max \left\{\max _{x \in \Omega_{1}} \operatorname{dist}\left(x, \Omega_{2}\right), \max _{x \in \Omega_{2}} \operatorname{dist}\left(x, \Omega_{1}\right)\right\} .
$$

That a closed convex cone in $\mathbb{R}^{n}$ is nontrivial simply means that it is different from the singleton $\{\mathbf{0}\}$ and different from the whole space $\mathbb{R}^{n}$.

Proposition 2.1 The function $T: P^{c} \rightarrow \Xi\left(\mathbb{R}^{n}\right)$ is continuous. In fact, for all $z_{1}, z_{2} \in P^{c}$, one has

$$
\varrho\left(T\left(z_{1}\right), T\left(z_{2}\right)\right) \leq \frac{2}{\operatorname{dist}\left(z_{1}, P\right)+\operatorname{dist}\left(z_{2}, P\right)}\left\|z_{1}-z_{2}\right\| .
$$

Proof It is not difficult to check that

$$
\begin{array}{ll}
T(z) \subseteq \liminf _{v \rightarrow z} T(v) & \text { for all } z \in \mathbb{R}^{n}, \\
T(z) \supseteq \limsup _{v \rightarrow z} T(v) & \text { for all } z \in P^{c},
\end{array}
$$

where the lower and upper limits are understood in the Painlevé-Kuratowski sense. This proves already the continuity of $T: P^{c} \rightarrow \Xi\left(\mathbb{R}^{n}\right)$, because convergence in $\Xi\left(\mathbb{R}^{n}\right)$ with respect to the metric $\varrho$ is equivalent to Painlevé-Kuratowski set-convergence. However, one can go beyond mere continuity and prove a Lipschitz type estimate like (6). Let us examine the expression

$$
e\left(K_{1}, K_{2}\right):=\max _{x \in K_{1} \cap \mathbb{S}_{n}} \operatorname{dist}\left(x, K_{2} \cap \mathbb{S}_{n}\right)
$$

for $K_{1}=R_{+}\left(\Omega_{1}\right)$ and $K_{2}=R_{+}\left(\Omega_{2}\right)$, where $\Omega_{1}, \Omega_{2} \subseteq \mathbb{R}^{n}$ are nonempty convex compact sets not containing the origin $\mathbf{0}$. A direct computation shows that

$$
e\left(K_{1}, K_{2}\right)=\max _{u_{1} \in \Omega_{1}} \min _{u_{2} \in \Omega_{2}}\left\|\frac{u_{1}}{\left\|u_{1}\right\|}-\frac{u_{2}}{\left\|u_{2}\right\|}\right\| .
$$

But the Dunkl-Williams inequality [12] asserts that

$$
\left\|\frac{u_{1}}{\left\|u_{1}\right\|}-\frac{u_{2}}{\left\|u_{2}\right\|}\right\| \leq 2 \frac{\left\|u_{1}-u_{2}\right\|}{\left\|u_{1}\right\|+\left\|u_{2}\right\|}
$$

for any pair $u_{1}, u_{2}$ of nonzero vectors. One gets in this way

$$
e\left(K_{1}, K_{2}\right) \leq \ell \max _{u_{1} \in \Omega_{1}} \min _{u_{2} \in \Omega_{2}}\left\|u_{1}-u_{2}\right\|
$$


with $\ell:=2\left[\operatorname{dist}\left(\mathbf{0}, \Omega_{1}\right)+\operatorname{dist}\left(\mathbf{0}, \Omega_{2}\right)\right]^{-1}$. A similar estimate holds for the symmetric term $e\left(K_{2}, K_{1}\right)$, so one ends up with

$$
\varrho\left(K_{1}, K_{2}\right) \leq \ell \text { haus }\left(\Omega_{1}, \Omega_{2}\right) .
$$

If one takes $\Omega_{j}=P-z_{j}$, then $\operatorname{dist}\left(\mathbf{0}, \Omega_{j}\right)=\operatorname{dist}\left(z_{j}, P\right)$ and $\operatorname{haus}\left(\Omega_{1}, \Omega_{2}\right)=\left\|z_{1}-z_{2}\right\|$. Substituting this information in (8) one gets (6).

The inequality (6) shows that $T$ is Lipschitzian on any compact set contained in $P^{c}$. Indeed, if $Z$ is the compact set under consideration, then

$$
\varrho\left(T\left(z_{1}\right), T\left(z_{2}\right)\right) \leq(1 / \beta)\left\|z_{1}-z_{2}\right\| \quad \text { for all } z_{1}, z_{2} \in Z
$$

with $\beta:=\min _{z \in Z} \operatorname{dist}(z, P)$.

Remark 2.2 The set $P^{c}$ is open in $\mathbb{R}^{n}$. If $\left\{z_{k}\right\}_{k \in \mathbb{N}}$ is a sequence in $P^{c}$ converging to $\bar{z} \in \operatorname{bd}(P)$, then it does not follow necessarily that $\lim _{k \rightarrow \infty} \varrho\left(T\left(z_{k}\right), T(\bar{z})\right)=0$. In other words, is not always possible to predict the behavior of $T(z)$ as $z$ approaches to a point on the boundary of $P$. This issue is discussed at length in [21, Section 5].

2.2 Existence of solutions for the illumination problem

The optimal value of the illumination problem (2) is denoted by $s_{*}$. The feasible set

$$
\begin{aligned}
W & :=\left\{(z, y, s) \in Z \times \mathbb{S}_{n} \times[0,1]: A \subseteq C(z, y, s)\right\} \\
& =\left\{(z, y, s) \in Z \times \mathbb{S}_{n} \times[0,1]: f_{i}(z, y, s) \leq 0 \text { for all } i \in I\right\}
\end{aligned}
$$

of (2) is closed, but nonconvex in general. For making sure that $W$ is nonempty one asks $Z$ to contain a point that is not in the interior of $P$.

Proposition 2.3 The set $W$ is nonempty if and only if $Z$ is not included in $\operatorname{int}(P)$.

Proof For $(z, y, s) \in Z \times \mathbb{S}_{n} \times[0,1]$ one has

$$
\begin{aligned}
A \subseteq C(z, y, s) & \Leftrightarrow A-z \subseteq \Gamma(y, s) \\
& \Leftrightarrow P-z \subseteq \Gamma(y, s) \\
& \Leftrightarrow T(z) \subseteq \Gamma(y, s) .
\end{aligned}
$$

Hence, the illumination problem (2) is feasible if and only if $T(z) \neq \mathbb{R}^{n}$ for some $z \in Z$. In other words, $W$ is nonempty if and only if there exists a point $z \in Z$ that is not in the interior of $P$.

The next proposition shows that the $z$-component of a solution to the illumination problem (2) is necessarily on the boundary of $Z$.

Proposition 2.4 Assume that $A$ is not on ray emanating from a point of $Z$. Let $(z, y, s)$ be a solution to (2). Then

$$
Z \cap \operatorname{int}[z-\Gamma(y, s)]=\emptyset .
$$

In particular, $z \in \mathrm{bd}(Z)$. 
Proof Since $A$ is not on ray emanating from a point of $Z$, the sharpness coefficient $s$ is smaller than 1. Consequently, $\Gamma(y, s)$ has nonempty interior. Suppose that (9) is not true. Then there exists $w \in \operatorname{int}[\Gamma(y, s)]$ such that $z_{0}:=z-w$ belongs to $Z$. Since $A-z \subseteq \Gamma(y, s)$ and

$$
\Gamma(y, s)+\operatorname{int}[\Gamma(y, s)]=\operatorname{int}[\Gamma(y, s)]
$$

one obtains

$$
A-z_{0}=(A-z)+w \subseteq \operatorname{int}[\Gamma(y, s)] .
$$

In other words, for all $i \in I$, one can write

$$
a_{i}-z_{0} \in \operatorname{int}[\Gamma(y, s)] .
$$

The interiority condition (10) implies that each $a_{i}$ is different from $z_{0}$. Moreover,

$$
a_{i}-z_{0} \in \operatorname{bd}\left[\Gamma\left(y, s_{i}\right)\right] \subseteq \Gamma\left(y, s_{i}\right) \subseteq \Gamma(y, \tilde{s}),
$$

where $\tilde{s}:=\min _{i \in I} s_{i}$ and

$$
0 \leq s<s_{i}:=\left\langle\frac{a_{i}-z_{0}}{\left\|a_{i}-z_{0}\right\|}, y\right\rangle \leq 1 .
$$

Note that the sharpness coefficient $\tilde{s}$ is larger than $s$ and that

$$
A \subseteq z_{0}+\Gamma(y, \tilde{s})=C\left(z_{0}, y, \tilde{s}\right) .
$$

This contradicts the maximality of $s$.

The next lemma provides an easily computable lower bound for $s_{*}$. The symbols $c_{A}$ and $r_{A}$ indicate, respectively, the Chebishev center and the Chebishev radius of the sample $A$. By definition, $\left(c_{A}, r_{A}\right)$ is the unique solution to

$$
\left\{\begin{array}{l}
\operatorname{minimize} r \\
x \in \mathbb{R}^{n}, r \geq 0 \\
A \subseteq x+r \mathbb{B}_{n},
\end{array}\right.
$$

where $\mathbb{B}_{n}:=\left\{x \in \mathbb{R}^{n}:\|x\| \leq 1\right\}$ is the $n$-dimensional closed unit ball.

Lemma 2.5 For any $z \in Z$ that is not in the Chebishev ball $c_{A}+r_{A} \mathbb{B}_{n}$, one can write

$$
\left[1-\left(\frac{r_{A}}{\left\|z-c_{A}\right\|}\right)^{2}\right]^{1 / 2} \leq s_{*} .
$$

Proof The Chebishev ball contains the sample $A$. In fact, it is the closed ball of smallest radius that contains $A$. It follows that

$$
A-z \subseteq M(y, r):=\mathbb{R}_{+}\left(y+r \mathbb{B}_{n}\right),
$$

where $y:=\left\|c_{A}-z\right\|^{-1}\left(c_{A}-z\right)$ and $r:=\left\|c_{A}-z\right\|^{-1} r_{A}$. A matter of computation shows that

$$
M(y, r)=\Gamma(y, s)
$$

with $s=\left[1-r^{2}\right]^{1 / 2}$. The formula (13) can be found for instance in [18, Lemma 2.1]. The combination of (12) and (13) yields the inequality (11). 
The issue concerning the existence of solutions to illumination problem (2) is settled in Theorem 2.6. The proof of the part (a) is based on Lemma 2.5, but one could use also a more direct argument.

Theorem 2.6 Suppose that $A$ is not on ray emanating from a point of $Z$.

(a) If $Z$ is unbounded, then $s_{*}=1$ and (2) has no solution.

(b) If $Z$ is bounded and not included in the interior of $P$, then (2) has at least one solution.

Proof Suppose that $Z$ is unbounded. Then there exists a sequence $\left\{z_{k}\right\}_{k \in \mathbb{N}}$ in $Z$ such that $\lim _{k \rightarrow \infty}\left\|z_{k}\right\|=\infty$. Lemma2.5 implies that

$$
\left[1-\left(\frac{r_{A}}{\left\|z_{k}-c_{A}\right\|}\right)^{2}\right]^{1 / 2} \leq s_{*}
$$

for all $k$ sufficiently large. By passing to the limit in (14) one gets $s_{*}=1$. Supposed now that $Z$ is bounded and not included in the interior of $P$. In such a case the problem (2) is about maximizing a linear function on a nonempty compact set.

2.3 Link with the theory of incenters and circumcenters

As explained next, the illumination problem (2) is somewhat related with the problem of finding the circumcenter of a polyhedral convex cone. Our first observation is that

$$
s_{*}=\sup _{z \in Z} \Phi(z),
$$

where $\Phi(z)$ denotes the optimal value of the lower dimensional problem

$$
(\mathcal{P})_{z} \quad\left\{\begin{array}{l}
\text { maximize } s \\
(y, s) \in \mathbb{S}_{n} \times[0,1] \\
f_{i}(z, y, s) \leq 0 \text { for all } i \in I
\end{array}\right.
$$

The solution set to (15) is given by

$$
\begin{aligned}
Z_{*} & :=\left\{z \in Z: \Phi(z)=s_{*}\right\} \\
& =\{z:(z, y, s) \text { solves (2) }\} .
\end{aligned}
$$

For a fixed $z \in Z$, the reduced problem (16) has a very interesting geometric interpretation. Note that (16) can be rewritten as

$$
\left\{\begin{array}{l}
\operatorname{maximize} s \\
(y, s) \in \mathbb{S}_{n} \times[0,1] \\
T(z) \subseteq \Gamma(y, s)
\end{array}\right.
$$

In other words, one must find a standard revolution cone of smallest aperture angle that contains the polyhedral cone $T(z)$. The optimization problem (17) has been studied in [16, $17,19]$ in the more general context in which $T(z)$ is changed by an arbitrary $K \in \Xi\left(\mathbb{R}^{n}\right)$. The optimal value of

$$
\left\{\begin{array}{l}
\operatorname{maximize} s \\
(y, s) \in \mathbb{S}_{n} \times[0,1] \\
K \subseteq \Gamma(y, s)
\end{array}\right.
$$

is denoted by $\tau(K)$ and called the sharpness coefficient of $K$ (cf. [19, Section 5]). If $K$ is pointed, then the maximization problem (18) admits exactly one solution. The $y$-component of such solution is called the circumcenter of $K$. 
Theorem 2.7 For all $z \in P^{c}$, the term $\Phi(z)$ is equal to the sharpness coefficient of $T(z)$. In particular, the function $\Phi: P^{c} \rightarrow \mathbb{R}$ is not merely continuous but satisfies

$$
\left|\Phi\left(z_{1}\right)-\Phi\left(z_{1}\right)\right| \leq \frac{2}{\operatorname{dist}\left(z_{1}, P\right)+\operatorname{dist}\left(z_{2}, P\right)}\left\|z_{1}-z_{2}\right\|
$$

for all $z_{1}, z_{2} \in P^{c}$.

Proof For all $z \in P^{c}$, the cone $T(z)$ is pointed. Recall that $\Phi(z)$ is equal to the optimal value of (17), so the first part of the theorem is nothing new. For proving the last part of the theorem we view $z \in P^{c} \mapsto \Phi(z)=\tau(T(z))$ as a composite function. Proposition 13 of [19] asserts that

$$
\left|\tau\left(K_{1}\right)-\tau\left(K_{1}\right)\right| \leq \varrho\left(K_{1}, K_{2}\right)
$$

for all $K_{1}, K_{2} \in \Xi\left(\mathbb{R}^{n}\right)$. One gets in this way

$$
\left|\Phi\left(z_{1}\right)-\Phi\left(z_{1}\right)\right| \leq \varrho\left(T\left(z_{1}\right), T\left(z_{2}\right)\right)
$$

for all $z_{1}, z_{2} \in P^{c}$. Proposition 2.1 completes the proof of (19).

The inequality (19) implies that $\Phi$ is Lipschitzian on any compact set contained in $P^{c}$. In particular, $\Phi: P^{c} \rightarrow \mathbb{R}$ is locally Lipschitz and one obtains the upper estimate

$$
\partial \Phi(z) \subseteq[\operatorname{dist}(z, P)]^{-1} \mathbb{B}_{n}
$$

for the Clarke subdifferential

$$
\partial \Phi(z):=\operatorname{co}\left\{\lim _{k \rightarrow \infty} \nabla \Phi\left(z_{k}\right):\left\{z_{k}\right\}_{k \in \mathbb{N}} \rightarrow z, z_{k} \in \operatorname{dom}(\nabla \Phi)\right\}
$$

of $\Phi$ at $z \in P^{c}$. In general, $\Phi: P^{c} \rightarrow \mathbb{R}$ is a nondifferentiable function, but the set $\operatorname{dom} \nabla \Phi$ of differentiability points of $\Phi$ has full Lebesgue measure in $P^{c}$.

Remark $2.8 \Phi$ is finite-valued also on the boundary of $P$. For all $z \in[\operatorname{int}(P)]^{c}$ one has

$$
\Phi(z)=\sup \{s:(y, s) \in M(z)\},
$$

where $M(z)$ is the nonempty compact set given by

$$
M(z):=\left\{(y, s) \in \mathbb{S}_{n} \times[0,1]: T(z) \subseteq \Gamma(y, s)\right\} .
$$

By using (7) and the continuity of the function $\Gamma: \mathbb{S}_{n} \times[0,1] \rightarrow \Xi\left(\mathbb{R}^{n}\right)$, one can check that $M$ is upper-semicontinuous as set-valued map between the metric spaces $[\operatorname{int}(P)]^{c}$ and $\mathbb{S}_{n} \times[0,1]$. By relying on Berge's maximum theorem (cf. [6, Theorem 1.4.16]), one deduces that $\Phi:[\operatorname{int}(P)]^{c} \rightarrow \mathbb{R}$ is upper-semicontinuous. However, $\Phi(z)$ may not converge to $\Phi(\bar{z})$ as $z$ approaches to a point $\bar{z}$ on the boundary of $P$.

Corollary 2.9 For all $z \in P^{c}$ one has

$$
\max \left\{0,1-\frac{\operatorname{diam}(A)}{\operatorname{dist}(z, P)}\right\} \leq \Phi(z),
$$

where $\operatorname{diam}(A)=\max _{i, j \in I}\left\|a_{i}-a_{j}\right\|$. In particular,

$$
\lim _{\|z\| \rightarrow \infty} \Phi(z)=1 .
$$


Proof The proof is inspired from Theorems 3.2 and 3.4 in [21]. Since the sharpness coefficient of a ray is equal to 1 , the nonexpansiveness of $\tau: \Xi\left(\mathbb{R}^{n}\right) \rightarrow \mathbb{R}$ yields

$$
|\Phi(z)-1| \leq \varrho\left(T(z), R_{+} y\right)
$$

for all $y \in \mathbb{S}_{n}$. If $y$ is a unit vector taken from $T(z)$, then

$$
\varrho\left(T(z), R_{+} y\right)=\max _{u \in T(z) \cap \mathbb{S}_{n}} \operatorname{dist}\left(u, R_{+} y \cap \mathbb{S}_{n}\right)=\max _{u \in T(z) \cap \mathbb{S}_{n}}\|u-y\| .
$$

This and (21) yield $1-\Phi(z) \leq \operatorname{diam}\left(T(z) \cap \mathbb{S}_{n}\right)$. By the Dunkl-Williams inequality, one has

$$
\begin{aligned}
\operatorname{diam}\left(T(z) \cap \mathbb{S}_{n}\right) & =\max _{u, v \in P}\left\|\frac{u-z}{\|u-z\|}-\frac{v-z}{\|v-z\|}\right\| \\
& \leq \frac{1}{\operatorname{dist}(z, P)} \max _{u, v \in P}\|u-v\| .
\end{aligned}
$$

So, we have proven that

$$
1-\Phi(z) \leq \frac{\operatorname{diam}(P)}{\operatorname{dist}(z, P)} .
$$

It suffices now to observe that $\operatorname{diam}(P)=\operatorname{diam}(A)$, because a polytope admits always a pair of vertices attaining its diameter.

A problem closely related to (18) is that of finding a largest ball centered at a unit vector and contained in a given $Q \in \Xi\left(\mathbb{R}^{n}\right)$ :

$$
\left\{\begin{array}{l}
\operatorname{maximize} r \\
(x, r) \in \mathbb{S}_{n} \times[0,1] \\
x+r \mathbb{B}_{n} \subseteq Q .
\end{array}\right.
$$

The optimal value of (22) is denoted by $\rho(Q)$ and called the inradius of $Q$. If the cone $Q$ has nonempty interior, then the maximization problem (22) admits exactly one solution. The $x$-component of such solution is called the incenter of $Q$.

Lemma 2.10 Suppose that $K \in \Xi\left(\mathbb{R}^{n}\right)$ is pointed or, equivalently, that its positive dual cone

$$
Q:=\left\{v \in \mathbb{R}^{n}:\langle v, u\rangle \geq 0 \text { for all } u \in K\right\}
$$

has nonempty interior. In such a case, $\tau(K)=\rho(Q)$ and the circumcenter of $K$ is equal to the incenter of $Q$.

Lemma 2.10 is borrowed from $[16,17]$. A matter of computation shows that the positive dual cone of $T(z)$ is equal to the polyhedral convex cone

$$
R(z):=\left\{x \in \mathbb{R}^{n}:\left\langle a_{i}-z, x\right\rangle \geq 0 \text { for all } i \in I\right\} .
$$

Hence, for all $z \in P^{c}$, the term $\Phi(z)$ can be seen also as the inradius of $R(z)$. For notational convenience, we introduce the vector function $\mathbf{y}: P^{c} \rightarrow \mathbb{R}^{n}$ given by

$$
\begin{aligned}
\mathbf{y}(z) & :=\text { circumcenter of } T(z) \\
& =\text { incenter of } R(z) .
\end{aligned}
$$

The next corollary is a direct consequence of Lemma 2.10 and does not need further explanation. 
Corollary 2.11 Let $Z$ be a compact set not intersecting $P$. Then

$$
(z, y, s) \text { solves }(2) \Leftrightarrow z \text { solves }(15) \text { and }(y, s)=(\mathbf{y}(z), \Phi(z)) .
$$

For each fixed $z \in P^{c}$, the pair $(\mathbf{y}(z), \Phi(z))$ is the unique optimal solution to (17). So, one can view the set $C(z, \mathbf{y}(z), \Phi(z))$ as the optimal conic light beam with prescribed apex $z$. We next explain how to evaluate the functions $\mathbf{y}(\cdot)$ and $\Phi(\cdot)$ in practice. In what follows, the notation $\Lambda_{m}$ refers to the standard simplex in $\mathbb{R}^{m}$.

Theorem 2.12 Let $m$ be the cardinality of I. Then $\Phi$ admits on $P^{c}$ the lower hull representation

$$
\Phi(z)=\min _{\lambda \in \Lambda_{m}}\left\|\sum_{i=1}^{m} \lambda_{i} \mathbf{c}_{i}(z)\right\|,
$$

where each $\mathbf{c}_{i}: P^{c} \rightarrow \mathbb{R}^{n}$ is given by

$$
\mathbf{c}_{i}(z):=\frac{a_{i}-z}{\left\|a_{i}-z\right\|} .
$$

In particular, if $Z$ is a compact set not intersecting $P$, then the optimal value of (2) admits the max-min formulation

$$
s_{*}=\max _{z \in Z} \min _{\lambda \in \Lambda_{m}}\left\|\sum_{i=1}^{m} \lambda_{i} \mathbf{c}_{i}(z)\right\| .
$$

Proof We take $z \in P^{c}$ and write (23) in the equivalent form

$$
R(z)=\left\{x \in \mathbb{R}^{n}:\left\langle\mathbf{c}_{i}(z), x\right\rangle \geq 0 \text { for all } i \in I\right\} .
$$

Note that each $a_{i}$ is different from $z$ and therefore the unit vector $\mathbf{c}_{i}(z)$ is well defined. The representation formula (24) is obtained by applying [15, Proposition 3.3] to the polyhedral convex cone (27). The second part of the theorem follows by combining (24) and (15).

Remark 2.13 One of the oldest illumination problem in the history of mathematics was formulated by the scholar Regiomontanus in the 15th century. Regiomontanus asked to find a point on the ground so that a perpendicularly suspended rod appears largest, i.e., subtends the greatest visual angle (cf.[11]). Regiomontanus' problem is a particular instance of the minimization problem

$$
\tilde{s}:=\min _{z \in Z} \Phi(z) .
$$

If $Z$ is a compact set not intersecting $P$, then one gets

$$
\tilde{s}=\min _{\substack{z \in Z \\ \lambda \in \Lambda_{m}}}\left\|\sum_{i=1}^{m} \lambda_{i} \mathbf{c}_{i}(z)\right\| .
$$

In principle, computing $\tilde{s}$ is simpler than computing $s_{*}$.

An interesting aspect of (24) is that $\Phi(z)$ can be evaluated by solving an "easy" convex optimization problem. To be more precise, one has to find the projection $\pi(z):=\operatorname{Proj}[\mathbf{0}, \Omega(z)]$ of $\mathbf{0}$ onto the polytope $\Omega(z):=\operatorname{co}\left\{\mathbf{c}_{1}(z), \ldots, \mathbf{c}_{m}(z)\right\}$. By relying on [15, Proposition 3.1], one can write

$$
\Phi(z)=\min _{i \in I}\left\langle\mathbf{c}_{i}(z), \mathbf{y}(z)\right\rangle
$$


with $\mathbf{y}(\cdot)$ admitting the explicit formula $\mathbf{y}(z)=\pi(z) /\|\pi(z)\|$. Note that (28) is yet another characterization of the function $\Phi: P^{c} \rightarrow \mathbb{R}$.

\subsection{Differentiability properties of $\Phi$}

The lower hull representation (24) and the continuity of

$$
(\lambda, z) \in \Lambda_{m} \times P^{c} \mapsto L(\lambda, z):=\left\|\sum_{i=1}^{m} \lambda_{i} \mathbf{c}_{i}(z)\right\|
$$

confirms that $\Phi$ is continuous on $P^{c}$. The function (29) is differentiable with respect to $z$ and its partial gradient is given by

$$
\nabla_{z} L(\lambda, z)=\left(\sum_{i=1}^{m} \frac{\lambda_{i}}{\left\|a_{i}-z\right\|}\left(c_{i}(z)\left(c_{i}(z)\right)^{T}-I_{n}\right)\right) \frac{\sum_{i=1}^{m} \lambda_{i} \mathbf{c}_{i}(z)}{\left\|\sum_{i=1}^{m} \lambda_{i} \mathbf{c}_{i}(z)\right\|},
$$

where $I_{n}$ is the identity matrix of order $n$ and the superscript " $T$ " stands for transposition. Beware that the differentiability of each $L(\lambda, \cdot)$ does not imply the differentiability of $\Phi$. In fact, it is not difficult to construct a sample $A$ for which $\Phi: P^{c} \rightarrow \mathbb{R}$ is nondifferentiable. The next theorem shows that $\Phi$ is directionally differentiable and Clarke lower-regular.

Theorem 2.14 Let $m$ be the cardinality of I. Then

(a) $\Phi: P^{c} \rightarrow \mathbb{R}$ is directionally differentiable and its directional derivative at $z \in P^{c}$ is given by

$$
h \in \mathbb{R}^{n} \mapsto \Phi^{\prime}(z ; h)=\min _{\lambda \in \Lambda(z)}\left\langle\nabla_{z} L(\lambda, z), h\right\rangle,
$$

where $L$ is the function introduced in (29) and $\Lambda(z)$ is the solution set to (24).

(b) $\Phi: P^{c} \rightarrow \mathbb{R}$ is Clarke lower-regular, i.e., for all $z \in P^{c}$ the directional derivative $\Phi^{\prime}(z ; \cdot)$ coincides with the Clarke lower derivative

$$
h \in \mathbb{R}^{n} \mapsto \Phi^{\downarrow}(z ; h):=\liminf _{\substack{v \rightarrow z \\ t \rightarrow 0^{+}}} \frac{\Phi(v+t h)-\Phi(v)}{t} .
$$

In particular, the Clarke subdifferential of $\Phi$ admits the representation

$$
\partial \Phi(z)=\operatorname{co}\left\{\nabla_{z} L(\lambda, z): \lambda \in \Lambda(z)\right\} .
$$

Proof For proving (a) it suffices to apply Danskin's directional differentiability theorem [10] to

$$
z \in P^{c} \mapsto \Phi(z)=\min _{\lambda \in \Lambda_{m}} L(\lambda, z) .
$$

The set $\Lambda_{m}$ is compact and the function $(\lambda, z) \in \Lambda_{m} \times P^{c} \mapsto \nabla_{z} L(\lambda, z)$ is continuous. Hence, the hypotheses of Danskin's theorem are in force. Part (b) is obtained by applying [8, Theorem 2.1].

The formula (30) shows that $\Phi$ is differentiable at each $z \in P^{c}$ for which (31) is a singleton. For instance, if the vectors $\mathbf{c}_{1}(z), \ldots, \mathbf{c}_{m}(z)$ are linearly independent, then the optimization problem (24) admits a unique solution, say $\lambda(z)$, and, in such a case, one gets $\nabla \Phi(z)=\nabla_{z} L(\lambda(z), z)$. Linear independence of the $\mathbf{c}_{i}(z)$ 's is however a very stringent assumption, because usually the size of the sample $A$ is much larger than the dimension of the underlying space. 
Corollary 2.15 For all $z \in P^{c}$, the directional derivative $\Phi^{\prime}(z ; \cdot): \mathbb{R}^{n} \rightarrow \mathbb{R}$ is concave and

$$
\max _{\|h\|=1}\left|\Phi^{\prime}(z ; h)\right| \leq \frac{1}{\operatorname{dist}(z, P)} .
$$

Proof The first part follows from Theorem 2.14. The estimate (32) is a consequence of (19).

\subsection{Directions of ascent for the function $\Phi$}

By Proposition 2.4 one knows that all the solutions to the maximization problem (15) are on the boundary of $Z$. As shown in the proposition below, the interior of $Z$ is also free of local solutions to (15).

Proposition 2.16 Let $Z$ be a compact set not intersecting $P$. Assume that $A$ is not on ray emanating from a point of $Z$. Then any local maximum of $\Phi: Z \rightarrow \mathbb{R}$ is on the boundary of $Z$.

Proof Let $z \in P^{c}$. Recall that $\mathbf{y}(z)$ stands for the circumcenter of $T(z)$. One has

$$
\Phi(z-t \mathbf{y}(z))>\Phi(z)
$$

for all positive real $t$. The above inequality is obtained by working out the proof of Proposition 2.4 with the particular choice $w=t \mathbf{y}(z)$. Now, if $z \in \operatorname{int}(Z)$, then $z-t \mathbf{y}(z)$ remains in $Z$ for all $t$ sufficiently small. Hence, a point $z$ in the interior of $Z$ cannot be a local maximum of $\Phi: Z \rightarrow \mathbb{R}$.

The inequality (33) says that $-\mathbf{y}(z)$ is a direction of ascent of $\Phi$ at the point $z$. This information could be useful for algorithmic purposes. It is tempting to conjecture that $-\mathbf{y}(z)$ is a steepest ascent direction, but we have no evidence supporting this conjecture. The next proposition shows that $\Phi(z)$ does not decrease if the argument of $\Phi$ moves in a direction opposite to $T(z)$.

Proposition 2.17 Let $z \in P^{c}$ and $h \in T(z)$. Then for all positive real t one has

$$
\Phi(z-t h) \geq \Phi(z) .
$$

Proof We start by proving a result that is of interest by itself:

$$
T(z-w) \subseteq T(z) \text { for all } w \in T(z) .
$$

The statement (35) is somewhat implicit in [21, Lemma 5.1], but we give here a direct proof. Take

$$
w=\alpha(x-z) \quad \text { and } \quad u=\beta(v-(z-w))
$$

with $\alpha, \beta \geq 0$ and $x, v \in P$. One must prove that $u \in T(z)$. Let $\beta \neq 0$, otherwise $u=\mathbf{0}$ and we are done. Note that

$$
(1 / \beta) u=v-(z-\alpha(x-z))=v+\alpha x-(1+\alpha) z .
$$

Hence,

$$
\frac{1}{\beta(1+\alpha)} u=\left[\frac{1}{(1+\alpha)} v+\frac{\alpha}{(1+\alpha)} x\right]-z
$$

belongs to $P-z$, because the term between brackets is a convex combination of vectors in $P$. This shows that $u \in T(z)$ and completes the proof of (35). Now, since $T(z)$ is a cone, one can apply (35) to $w=t h$. This yields $T(z-t h) \subseteq T(z)$, from where one derives (34). 
We state next a sort of "strict" version of Proposition 2.17. To do this, we need first to prove a technical lemma concerning the function $\tau: \Xi\left(\mathbb{R}^{n}\right) \rightarrow \mathbb{R}$. It is plain to see that $\tau(\cdot)$ is nonincreasing with respect to set inclusion. The following property is a bit more subtle, but not altogether surprising.

Lemma 2.18 Let $K_{1}, K_{2} \in \Xi\left(\mathbb{R}^{n}\right)$ be such that $K_{1} \cap \mathbb{S}_{n} \subseteq \operatorname{int}\left(K_{2}\right)$. Then $\tau\left(K_{1}\right)>\tau\left(K_{2}\right)$.

Proof Let $(\bar{y}, \bar{s})$ be a solution to (18) for the choice $K=K_{2}$. Then $\bar{s}=\tau\left(K_{2}\right)$ and $K_{2} \subseteq$ $\Gamma(\bar{y}, \bar{s})$. It follows that $x \in \operatorname{int}(\Gamma(\bar{y}, \bar{s}))$ for all $x \in K_{1} \cap \mathbb{S}_{n}$. In other words, $\bar{s}<\langle\bar{y}, x\rangle$ for all $x \in K_{1} \cap \mathbb{S}_{n}$. If one sets

$$
\psi(\bar{y}):=\min _{x \in K_{1} \cap \mathbb{S}_{n}}\langle\bar{y}, x\rangle
$$

then $\psi(\bar{y})>\bar{s}$ and $K_{1} \subseteq \Gamma(\bar{y}, \psi(\bar{y}))$. Thus, $\tau\left(K_{1}\right) \geq \psi(\bar{y})>\bar{s}=\tau\left(K_{2}\right)$. This completes the proof.

Proposition 2.19 Suppose that $P$ has nonempty interior. Let $z \in P^{c}$ and $h \in \operatorname{int}(T(z))$. Then for all positive real $t$ one has

$$
\Phi(z-t h)>\Phi(z) .
$$

Proof For all $z \in P^{c}$ one has

$$
\operatorname{int}(T(z))=\{\alpha(x-z): \alpha>0, x \in \operatorname{int}(P)\} .
$$

In view of Lemma 2.18, for proving (38) it is enough to check that

$$
T(z-w) \cap \mathbb{S}_{n} \subseteq \operatorname{int}(T(z)) \text { for all } w \in \operatorname{int}(T(z)) .
$$

The above condition corresponds to a strict version of (35). Let $w \in \operatorname{int}(T(z))$ and $u$ be a unit vector in $T(z-w)$. One can represent $w$ and $u$ as in (36), with $\alpha>0, \beta>0, x \in \operatorname{int}(P)$, and $v \in P$. The term between brackets in (37) belongs now to the interior of $P$. Hence, $u \in \operatorname{int}(T(z))$.

Remark 2.20 Let $z \in P^{c}$. Beware that $\mathbf{y}(z)$ belongs to $T(z)$, but not necessarily to int $(T(z))$. So, the condition (33) cannot be inferred directly from Proposition 2.19.

2.6 How many $\mathbf{a}_{\mathbf{i}}$ 's are on the boundary of an optimal conic light beam?

We open a parenthesis and address the question raised above. We do this just out of curiosity. The next proposition shows that at least two points of the sample $\left\{a_{i}: i \in I\right\}$ must be on the boundary of the optimal conic light beam. The following lemma is intuitively clear.

Lemma 2.21 Let $m \geq 2$. Consider a polyhedral convex cone

$$
K:=\left\{\sum_{i=1}^{m} \mu_{i} c_{i}: \mu_{1} \geq 0, \ldots, \mu_{m} \geq 0\right\}
$$

generated by a finite collection $\left\{c_{i}\right\}_{i=1}^{m}$ of unit vectors. Suppose that $K$ is pointed and let $\left(y_{K}, s_{K}\right)$ be the solution to (18). Then the boundary

$$
E\left(y_{K}, s_{K}\right)=\left\{x \in \mathbb{R}^{n}: s_{K}\|x\|=\left\langle y_{K}, x\right\rangle\right\}
$$

of the revolution cone $\Gamma\left(y_{K}, s_{K}\right)$ contains at least two of the $c_{i}$ 's. 
Proof We suppose that $s_{K}<1$, otherwise $\Gamma\left(y_{K}, s_{K}\right)$ is a ray and we are done. If the $c_{i}$ 's are all in the interior of $\Gamma\left(y_{K}, s_{K}\right)$, then there exists $\left.\left.\tilde{s} \in\right] s_{K}, 1\right]$ such that $\left\{c_{1}, \ldots c_{m}\right\} \subseteq \Gamma\left(y_{K}, \tilde{s}\right)$, contradicting the maximality of $s_{K}$. Hence, at least one of the $c_{i}$ 's is in $E\left(y_{K}, s_{K}\right)$. Suppose for instance that

$$
\begin{aligned}
c_{1} & \in E\left(y_{K}, s_{K}\right), \\
\left\{c_{2}, \ldots, c_{m}\right\} & \subseteq \operatorname{int}\left[\Gamma\left(y_{K}, s_{K}\right)\right] .
\end{aligned}
$$

We must arrive to a contradiction. For $t \in] 0,1]$, consider the new axis

$$
y_{t}=\frac{y_{K}+t\left(c_{1}-y_{K}\right)}{\left\|y_{K}+t\left(c_{1}-y_{K}\right)\right\|}
$$

and the new sharpness coefficient $s_{t}=\left\langle c_{1}, y_{t}\right\rangle$. By construction, one has $c_{1} \in E\left(y_{t}, s_{t}\right)$. Since

$$
\begin{aligned}
& \lim _{t \rightarrow 0^{+}} y_{t}=y_{K} \\
& \lim _{t \rightarrow 0^{+}} s_{t}=\left\langle c_{1}, y_{K}\right\rangle=s_{K}\left\|c_{1}\right\|=s_{K},
\end{aligned}
$$

the interiority condition (39) ensures the existence of $\tilde{t} \in] 0,1]$ such that $\left\{c_{2}, \ldots c_{m}\right\} \subseteq$ $\Gamma\left(y_{t}, s_{t}\right)$ for all $\left.\left.t \in\right] 0, \tilde{t}\right]$. We have proven in this way that $K \subseteq \Gamma\left(y_{t}, s_{t}\right)$ whenever $t$ is sufficiently small. On the other hand, if one computes the right-derivative at 0 of the function $t \mapsto s_{t}$, then one gets

$$
\left(\frac{d s_{t}}{d t}\right)_{t=0^{+}}=1-\left\langle c_{1}, y_{K}\right\rangle>0 .
$$

Hence, $s_{t}>s_{K}$ for all $t$ near 0 , contradicting again the maximality of $s_{K}$.

As a by-product of Lemma 2.21 one gets:

Proposition 2.22 Let $Z$ be a compact set not intersecting $P$. Let $(z, y, s)$ be a solution to the illumination problem (2). Then the boundary of $C(z, y, s)$ contains at least two of the $a_{i}$ 's.

Proof Let the $\mathbf{c}_{i}(z)$ 's be defined as in (25). Note that $T(z)=\operatorname{cone}\left\{\mathbf{c}_{i}(z): i \in I\right\}$ is pointed and that $(y, s)$ is a solution to the problem (17). Lemma 2.21 implies that $E(y, s)$ contains at least two of the $\mathbf{c}_{i}(z)$ 's. In other words,

$$
\operatorname{bd}[C(z, y, s)]=\left\{x \in \mathbb{R}^{n}: s\|x-z\|-\langle y, x-z\rangle=0\right\}=z+E(y, s)
$$

contains at least two of the $a_{i}$ 's.

\subsection{Some particular cases}

This short section displays a few useful examples and counter-examples.

Example 2.23 If the $a_{i}$ 's are arranged on a line, then one can drop the intermediate points and keep only the two extreme points. Renumbering the $a_{i}$ 's if necessary, one may suppose that the extreme points are $a_{1}$ and $a_{2}$. Hence, $P$ is the closed line segment $\left[a_{1}, a_{2}\right]$ and the function $\Phi: P^{c} \rightarrow \mathbb{R}$ is given by

$$
\Phi(z)=\min _{\alpha \in[0,1]}\left\|(1-\alpha) \mathbf{c}_{1}(z)+\alpha \mathbf{c}_{2}(z)\right\| .
$$


The above minimum is attained at $\alpha=1 / 2$ and one gets the explicit formula

$$
\Phi(z)=\frac{1}{\sqrt{2}}\left[1+\left\langle\mathbf{c}_{1}(z), \mathbf{c}_{2}(z)\right\rangle\right]^{1 / 2} .
$$

The behavior of (40) is problematic if $z$ approaches either to $a_{1}$ or to $a_{2}$. If $Z$ is a compact set not intersecting $P$, then an optimal apex $z_{*} \in Z$ is found by solving the maximization problem

$$
\kappa_{*}:=\max _{z \in Z}\left\langle\mathbf{c}_{1}(z), \mathbf{c}_{2}(z)\right\rangle .
$$

A corresponding optimal axis $y_{*} \in \mathbb{S}_{n}$ is obtained by normalizing the vector

$$
\xi_{*}:=\frac{\mathbf{c}_{1}\left(z_{*}\right)+\mathbf{c}_{2}\left(z_{*}\right)}{2}
$$

and the optimal sharpness coefficient is given by $s_{*}=\left[\left(1+\kappa_{*}\right) / 2\right]^{1 / 2}$.

It is a great advantage to count on an explicit formula for $\Phi$ as in (40). What happens, however, if the points $a_{i}$ are not on a line? The planar case is discussed in the example below.

Example 2.24 Consider an arbitrary sample $\left\{a_{i}: i \in I\right\}$ on the plane. What makes the planar case so simple is that, for each $z \in P^{c}$, the supporting cone $T(z)$ is already a revolution cone. If $\vartheta(z)$ denotes the aperture angle of $T(z)$, then clearly

$$
\vartheta(z)=\max _{\substack{i, j \in I \\ i \neq j}} \arccos \left\langle\mathbf{c}_{i}(z), \mathbf{c}_{j}(z)\right\rangle .
$$

Since the smallest revolution cone that contains $T(z)$ is the cone $T(z)$ itself, one has

$$
\Phi(z)=\cos \left(\frac{\vartheta(z)}{2}\right)=\left[\frac{1+\cos (\vartheta(z))}{2}\right]^{1 / 2} .
$$

After simplification one ends up with

$$
\Phi(z)=\frac{1}{\sqrt{2}}\left[1+\min _{\substack{i, j \in I \\ i \neq j}}\left\langle\mathbf{c}_{i}(z), \mathbf{c}_{j}(z)\right\rangle\right]^{1 / 2}
$$

for all $z \in P^{c}$. Beware that the explicit formula (41) is no longer true for illumination problems in dimension three or more. In the nonplanar case, the expression on the right-hand side of (41) is a rather coarse upper bound of $\Phi(z)$.

The next example shows that the number of optimal apices in a planar illumination problem is not necessarily finite. In fact, it may form a continuum. This phenomenon is well known in the literature.

Example 2.25 Consider a sample formed with two points on the plane, say $a_{1}=(0,0)$ and $a_{2}=(1,0)$. Let

$$
Z_{\varepsilon}=\{(r \cos \theta, r \sin \theta): \theta \in[\varepsilon, \pi / 2] \text { and } r=\cos \theta+\sin \theta\},
$$

where $\varepsilon$ is a small positive parameter. Clearly, $Z_{\varepsilon}$ is a compact set not intersecting the line segment $\left[a_{1}, a_{2}\right]$. A direct computation shows that $\left\langle\mathbf{c}_{1}(z), \mathbf{c}_{2}(z)\right\rangle=1 / \sqrt{2}$ for all $z \in Z_{\varepsilon}$. Hence, the function $\Phi$ is constant over $Z_{\varepsilon}$. Every element of $Z_{\varepsilon}$ is therefore an optimal apex. If one sets $\varepsilon=0$, then the situation is of course entirely different. Such choice of $\varepsilon$ leads to an illumination problem admitting $z_{*}=a_{2}$ as unique optimal apex. 
The most bothersome aspect of the optimization problem (15) is the lack of convexity of the objective function $\Phi$. The nondifferentiability of $\Phi$ is not truly a serious drawback. The proposition below is part of the folklore of constrained optimization theory.

Proposition 2.26 Let $Z$ be a convex compact set not intersecting $P$. If $z_{*}$ is a local solution to $(15)$, then $z_{*}$ satisfies the criticality condition

$$
\Phi^{\prime}\left(z_{*} ; h\right) \leq 0 \quad \text { for all } h \in \mathcal{T}_{Z}\left(z_{*}\right)
$$

where $\mathcal{T}_{Z}\left(z_{*}\right)$ denotes the tangent cone to $Z$ at $z_{*}$.

A point $z_{*} \in Z$ as in (42) is called a critical point of (15). If $\Phi$ is differentiable at $z_{*}$, then the condition (42) takes the simpler form

$$
\nabla \Phi\left(z_{*}\right) \in \mathcal{N}_{Z}\left(z_{*}\right)
$$

where $\mathcal{N}_{Z}\left(z_{*}\right)$ denotes the normal cone to $Z$ at $z_{*}$. The reader should be aware that the optimization problem (15) may have critical points that are not a solution to (15), not even a local solution. This issue is illustrated with the help of the next example.

Example 2.27 Let $A$ be formed with $a_{1}=(1,0)$ and $a_{2}=(0,1)$. Consider $Z=$ $[-1,0] \times[-1,0]$, which is a convex compact set not intersecting $P$. Let us examine $\kappa(z)=\left\langle\mathbf{c}_{1}(z), \mathbf{c}_{2}(z)\right\rangle$ around $z_{*}=(-1,0)$. If $\alpha$ and $\beta$ are the Cartesian coordinates of $z$, then

$$
\kappa(z)=\kappa(\alpha, \beta)=\frac{\alpha^{2}+\beta^{2}-\alpha-\beta}{\sqrt{(1-\alpha)^{2}+\beta^{2}} \sqrt{(1-\beta)^{2}+\alpha^{2}}},
$$

and a matter of computation shows that

$$
\frac{\partial \kappa}{\partial \alpha}(-1,0)<0 \text { and } \frac{\partial \kappa}{\partial \beta}(-1,0)=0 .
$$

Since $\sqrt{2} \Phi(\cdot)=\sqrt{1+\kappa(\cdot)}$, one has

$$
\begin{aligned}
& \frac{\partial \Phi}{\partial \alpha}(\alpha, \beta)=\frac{1}{2 \sqrt{2}} \frac{1}{\sqrt{1+\kappa(\alpha, \beta)}} \frac{\partial \kappa}{\partial \alpha}(\alpha, \beta) \\
& \frac{\partial \Phi}{\partial \beta}(\alpha, \beta)=\frac{1}{2 \sqrt{2}} \frac{1}{\sqrt{1+\kappa(\alpha, \beta)}} \frac{\partial \kappa}{\partial \beta}(\alpha, \beta) .
\end{aligned}
$$

Hence,

$$
\frac{\partial \Phi}{\partial \alpha}(-1,0)<0 \text { and } \frac{\partial \Phi}{\partial \beta}(-1,0)=0 .
$$

The gradient $\nabla \Phi\left(z_{*}\right)$ belongs to the boundary of $\mathcal{N}_{Z}\left(z_{*}\right)=\mathbb{R}_{-} \times \mathbb{R}_{+}$. In particular, $z_{*}$ satisfies the criticality condition (43). However, $z_{*}$ is neither a local maximum nor a local minimum of $\Phi$ on $Z$. Since $\Phi$ is a nonconvex function, criticality alone is not enough to guarantee optimality. In this example, the critical point $z_{*}$ is not even a local extremum. 


\section{Numerical resolution of the illumination problem}

This section concentrates on the numerical resolution of the illumination problem (2). We have adopted a simple structure for set $Z$, by defining it either as a box or as the parametric representation of a curve. We have focused on the max-min formulation

$$
s_{*}=\max _{z \in Z} \Phi(z)=\max _{z \in Z} \min _{\lambda \in \Lambda_{m}}\left\|\sum_{i=1}^{m} \lambda_{i} \mathbf{c}_{i}(z)\right\|,
$$

which has been tackled by resorting to a bundle type method (BM) suited for local minimization of nonconvex, nonsmooth functions in presence of linear constraints. The code used is NCVX which is based on the algorithm described in [14] and has been already used for solving classification and separation problems in [2-5]. NCVX is written in Fortran 77.

In addition we have tested a direct evaluation (DE) method which is merely based on a sampling of the feasible set $Z$ and on direct calculation of $\Phi(z)$. We remark that latter calculation, as explained in Sect. 2.3, requires to solve, at each tested point of $Z$, a least-norm problem on a polytope, a structured convex quadratic program widely studied in the literature (see [13,20,23]).

We have applied DE when $Z$ (or its boundary) can be parameterized with the help of a single parameter ranging on a compact interval. Suppose for instance that

$$
Z=\{\mathbf{z}(t): t \in[0,1]\},
$$

where $\mathbf{z}:[0,1] \rightarrow \mathbb{R}^{n}$ is a continuous function.

In such a case, the maximization problem (15) is nothing but a one dimensional optimization problem:

$$
s_{*}=\max _{t \in[0,1]} \Phi(\mathbf{z}(t)) .
$$

Note that (45) is about maximizing the continuous function $\Phi_{\mathbf{z}}(\cdot):=\Phi(\mathbf{z}(\cdot))$ on the interval $[0,1]$. DE is quite easy to implement and reads as follows.

- Step 0. Initialization. A tolerance parameter $\varepsilon>0$ is given. Choose two integers $N$ and $p_{\max }$. Set $p=1$ and $s_{*}=0$.

- Step 1. Set $s^{(p)}=0$.

For $k=1, \ldots, p N$

- set $t^{(k)}:=\frac{1}{2}\left(\frac{k-1}{p N}+\frac{k}{p N}\right)$ and $z^{(k)}:=\mathbf{z}\left(t^{(k)}\right)$,

- solve the least-norm problem

$$
\Phi_{\mathbf{z}}\left(t^{(k)}\right)=\min _{\lambda \in \Lambda_{m}}\left\|\sum_{i=1}^{m} \lambda_{i} \mathbf{c}_{i}\left(z^{(k)}\right)\right\| .
$$

If $\Phi_{\mathbf{z}}\left(t^{(k)}\right)>s^{(p)}$, then set $s^{(p)}=\Phi_{\mathbf{z}}\left(t^{(k)}\right)$.

\section{EndFor}

If $\left|s^{(p)}-s_{*}\right| \leq \varepsilon$, then STOP.

- Step 2. If $s^{(p)}>s_{*}$, then set $s_{*}=s^{(p)}$. Increase $p$ by one unit. If $p>p_{\max }$, then STOP. Go to Step 1.

For the numerical resolution of the least norm problem (46) we have used the function "DQPROG" (from IMSL Fortran Numerical Libraries) written in Fortran 77 for solving a 
quadratic programming problem subject to linear equality/inequality constraints. We have set $\varepsilon=10^{-4}, N=1000$ and $p_{\max }=3$.

Both the codes BM and DE have been run on a $1.66 \mathrm{GHz}$ Intel Core Duo, under Microsoft Windows XP operating system. For our experiments we have considered the following test problems:

- Dataset Nr1. For each dimension $n$, we have defined $A$, the set of points to be illuminated, as the set of the canonical vectors $\left\{e_{1}, \ldots, e_{n}\right\}$ of $\mathbb{R}^{n}$. The apex $z$ is constrained to belong to the feasible set $Z=[-1,0]^{n}$. In this case the global optimal solution $\left(z_{*}, s_{*}\right)$ is the point

$$
z_{*}=(-1, \ldots,-1), \quad s_{*}=(1+3 / n)^{-1 / 2}(1+1 / n) .
$$

- Dataset Nr2. The set $A$ is formed with random points $a_{1}, \ldots, a_{m}$ uniformly distributed on $[0,1]^{n}$. The set $Z$ is the line segment

$$
Z=[u, v]:=\{(1-t) u+t v: t \in[0,1]\},
$$

where $u$ and $v$ are in turn uniformly distributed on $[-1,0]^{n}$, so that $Z$ does not intersect the polytope $P$. We have tested the program for several combinations of $n$ and $m$.

- Dataset Nr3. The set $A$ is formed with random points $a_{1}, \ldots, a_{m}$ uniformly distributed on $[-1,1] \times[-1,1] \times\{0\}$. The set $Z$ is the ellipse

$$
Z=\{(\alpha \cos (2 \pi t), \beta \sin (2 \pi t), 1): t \in[0,1]\}
$$

in $\mathbb{R}^{3}$. The constants $\alpha$ and $\beta$ are uniformly distributed on $[0,1]$. Note that the ellipse $Z$ and the polytope $P$ are in parallel planes, so they do not intersect.

- Dataset Nr4. The set $A$ is formed with random points $a_{1}, \ldots, a_{m}$ uniformly distributed on $[-1,0]^{n}$. The set $Z$ is the moment curve

$$
Z=\left\{\left(t, t^{2}, \ldots, t^{n}\right): t \in[0,1]\right\}
$$

in $\mathbb{R}^{n}$. In the plane, the moment curve is a parabola, in the three-dimensional space it is a twisted cubic, and so on.

Dataset $\mathrm{Nr} 1$ has been tackled exclusively by means of BM, Dataset $\mathrm{Nr} 2$ by both $\mathrm{BM}$ and DE, while Datasets Nr3 and Nr4 just by DE. In the following we present the results we have obtained.

$\mathrm{BM}$ has been run 100 times on Dataset $\mathrm{Nr} 1$, for randomly chosen starting points $z_{0} \in Z$ and for $n \in\{2,3, \ldots, 10\}$. In Table 1 we report for each $n$, the known global optimal solution $s_{*}$, the average optimal value $\bar{s}$ provided by the program and the maximum error $\max _{i=1, \ldots, 100}\left(s_{*}-s_{i}\right)$, where $s_{i}$ is the optimal value of $s$ relative to the $i$-th experiment. We have considered a 20 digit representation for the variable $s$.

As for Dataset Nr2, we have considered all possible combinations of $m \in\{5,10,15,20\}$ and $n \in\{3,4,5\}$. For each combination $(m, n)$ we have generated 100 times the interval endpoints $u, v$. In Table 2 we report comparison of the results provided by BM and DE. For each combination $(m, n)$ we report the ordered list $\left(\bar{t}_{\mathrm{BM}}, \bar{t}_{\mathrm{DE}}, W_{\mathrm{BM}}, W_{\mathrm{DE}}\right)$, where $\bar{t}_{\mathrm{BM}}$ is the average CPU time for $\mathrm{BM}, \bar{t}_{\mathrm{DE}}$ is the average CPU time for DE, $W_{\mathrm{BM}}$ is the number of times $\mathrm{BM}$ has provided a better objective function value at stop, and $W_{\mathrm{DE}}$ is the number of times DE has provided a better objective function value at stop. The results of the numerical experiments indicate that the max-min formulation (26) provides an effective computational scheme for numerically solving the illumination problem (2).

On Datasets Nr3 and Nr4 we have applied DE for $m \in\{5,10,15,20\}$. Figures 1 and 2 show typical shapes of $\Phi_{\mathbf{z}}$ when $Z$ is an ellipse and a moment curve in $\mathbb{R}^{3}$. 
Table 1 BM on Dataset Nr1

\begin{tabular}{llll}
\hline$n$ & $s_{*}$ & $\bar{s}$ & $\max _{i=1, \ldots, 100}\left(s_{*}-s_{i}\right)$ \\
\hline 2 & 0.9486833 & 0.9486833 & 0.0 \\
3 & 0.9428090 & 0.9428090 & $1.0 \times 10^{-15}$ \\
4 & 0.9449112 & 0.9449112 & $1.6 \times 10^{-10}$ \\
5 & 0.9486833 & 0.9486833 & $1.1 \times 10^{-7}$ \\
6 & 0.9525793 & 0.9525793 & $1.0 \times 10^{-15}$ \\
7 & 0.9561829 & 0.9561829 & $1.0 \times 10^{-15}$ \\
8 & 0.9594032 & 0.9594032 & $1.0 \times 10^{-15}$ \\
9 & 0.9622504 & 0.9622504 & $1.0 \times 10^{-15}$ \\
10 & 0.9647638 & 0.9647638 & $1.0 \times 10^{-15}$ \\
\hline
\end{tabular}

Table 2 BM versus DE on Dataset $\mathrm{Nr} 2$

\begin{tabular}{llll}
\hline$m$ & $n=3$ & $n=4$ & $n=5$ \\
\hline 5 & $(0.0022,0.1075,89,11)$ & $(0.0019,0.1145,86,14)$ & $(0.0030,0.1228,88,12)$ \\
10 & $(0.0028,0.3131,89,11)$ & $(0.0036,0.3381,87,13)$ & $(0.0033,0.3589,88,12)$ \\
15 & $(0.0042,0.6719,88,12)$ & $(0.0027,0.6950,89,11)$ & $(0.0025,0.7116,91,9)$ \\
20 & $(0.0041,1.2427,87,13)$ & $(0.0034,1.2600,86,14)$ & $(0.0044,1.2850,93,7)$ \\
\hline
\end{tabular}

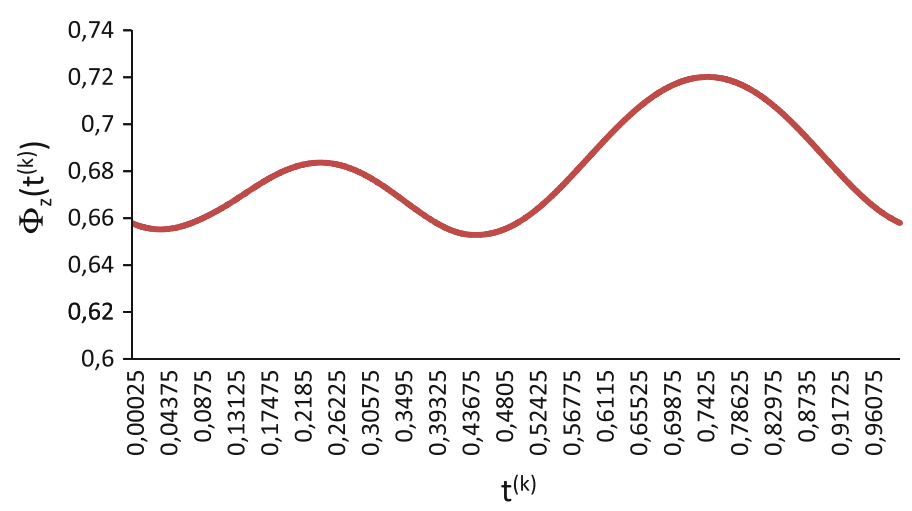

Fig. 1 A typical shape of $\Phi_{\mathbf{Z}}$ when $Z$ is an ellipse $(m=15)$

\subsection{The planar case}

The analysis of the illumination problem (15) is considerably easier in a two dimensional context. The particular case $n=2$ is mentioned only briefly in Example 2.24, but it has been the object of a discussion in [7]. Instead of recalling the specially tailored techniques developed in [7], we mention below a few useful guidelines for the resolution of (15) in the planar case. There are two interesting subcases for consideration:

I. Let $A=\left\{a_{1}, a_{2}\right\}$ and $Z$ be a polytope whose vertices $\left\{z_{k}\right\}_{k=1}^{r}$ are known. One assumes that the line passing through $a_{1}$ and $a_{2}$ does not intersect $Z$. As seen in Example 2.23, solving the illumination problem (15) amounts to maximize the function

$$
z \in Z \mapsto \kappa(z):=\left\langle\mathbf{c}_{1}(z), \mathbf{c}_{2}(z)\right\rangle .
$$




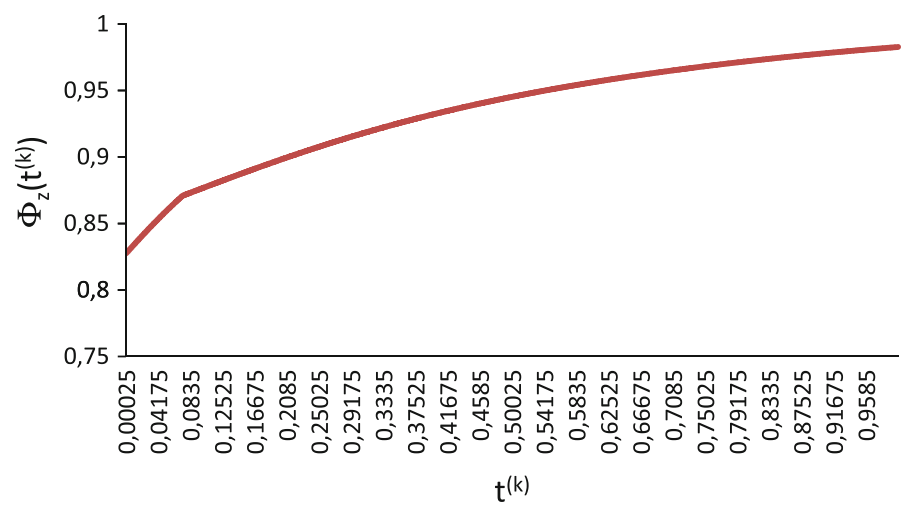

Fig. 2 A typical shape of $\Phi_{\mathbf{z}}$ when $Z$ is a moment curve in $\mathbb{R}^{3}(m=15)$

Thanks to Proposition 2.16, any maximum of (47) belongs to the boundary of $Z$. On the other hand, it is not difficult to show that (47) is a strictly quasiconvex function, i.e.,

$$
\kappa((1-t) u+t v)<\max \{\kappa(u), \kappa(v)\}
$$

if $t \in] 0,1[$ and $u, v \in Z$ are distinct. Hence, any maximum of (47) is in fact a vertex of $Z$. Thus, everything boils down to evaluate the function $\kappa(\cdot)$ at each $z_{k}$ and keep the vertex that yields the highest function value. Such numerical task can be performed in linear time in the number of vertices.

II. Let $Z$ be a polytope with vertices $\left\{z_{k}\right\}_{k=1}^{r}$ and $A=\left\{a_{i}\right\}_{i=1}^{m}$ be a sample with more than two points. One assumes that $Z$ does not intersect the convex hull of $A$. As seen in Example 2.24, solving (15) amounts to maximize

$$
z \in Z \mapsto \tilde{\kappa}(z):=\min _{1 \leq i<j \leq m}\left\langle\mathbf{c}_{i}(z), \mathbf{c}_{j}(z)\right\rangle .
$$

It may happen that no vertex of $Z$ maximizes (48), so one needs to evaluate $\tilde{\kappa}$ on the whole boundary of $Z$. Without loss of generality, we assume that $Z$ has nonempty interior and label the vertices of $Z$ in such a way that

$$
\operatorname{bd}(Z)=\bigcup_{k=1}^{r}\left[z_{k}, z_{k+1}\right]
$$

with $z_{r+1}:=z_{1}$. So, everything boils down to solve

$$
\operatorname{maximize}_{z \in\left[z_{k}, z_{k+1}\right]} \tilde{\kappa}(z)
$$

for each $k \in\{1, \ldots, r\}$ separately. Note that (49) can be treated for instance with the DE method.

\section{The penumbra problem}

For the penumbra problem (4) it is natural to assume that $Z$ is included in the interior of $P$, otherwise the problem admits a separating half-space as trivial solution. Of course, the hypothesis that $Z$ is closed remains always in force. 
Proposition 4.1 Assume that $Z \subseteq \operatorname{int}(P)$. Then the optimal value $s_{\diamond}$ of the problem (4) is positive and the solution set is nonempty. Furthermore, if $(z, y, s)$ is a solution to (4), then

$$
Z \cap \operatorname{int}[z+\Gamma(y, s)]=\emptyset .
$$

In particular, $z \in \operatorname{bd}(Z)$.

Proof The first part of the proposition is evident. Let $(z, y, s)$ be a solution to (4). Then $s=s_{\diamond}$ and

$$
s\left\|a_{i}-z\right\| \geq\left\langle a_{i}-z, y\right\rangle
$$

for all $i \in I$. Suppose that (50) is not true. Then there exists $w \in \operatorname{int}[\Gamma(y, s)]$ such that $z_{0}:=z+w$ belongs to $Z$. Since

$$
\begin{aligned}
& \left\|a_{i}-z_{0}\right\|=\left\|a_{i}-z-w\right\| \geq\left\|a_{i}-z\right\|-\|w\|, \\
& \left\langle a_{i}-z_{0}, y\right\rangle=\left\langle a_{i}-z-w, y\right\rangle=\left\langle a_{i}-z, y\right\rangle-\langle w, y\rangle,
\end{aligned}
$$

one obtains

$$
\begin{aligned}
s\left\|a_{i}-z_{0}\right\| & \geq s\left\|a_{i}-z\right\|-s\|w\| \\
& \geq\left\langle a_{i}-z, y\right\rangle-s\|w\|, \\
& \geq\left\langle a_{i}-z_{0}, y\right\rangle+\langle w, y\rangle-s\|w\| .
\end{aligned}
$$

But $\langle w, y\rangle-s\|w\|>0$, because $w$ belongs to the interior of $\Gamma(y, s)$. Hence,

$$
s\left\|a_{i}-z_{0}\right\|>\left\langle a_{i}-z_{0}, y\right\rangle
$$

for all $i \in I$. By setting $\tilde{s}:=\max _{i \in I} s_{i}$ with

$$
s_{i}:=\max \left\{0,\left\langle\frac{a_{i}-z_{0}}{\left\|a_{i}-z_{0}\right\|}, y\right\rangle\right\},
$$

one gets $0 \leq \tilde{s}<s$ and $A \subseteq D\left(z_{0}, y, \tilde{s}\right)$. This contradicts the minimality of $s$.

The next result is similar in spirit to Proposition 2.22.

Proposition 4.2 Let $Z \subseteq \operatorname{int}(P)$ and $(z, y, s)$ be a solution to the penumbra problem (4). Then the boundary of $D(z, y, s)$ contains at least two of the $a_{i}$ 's.

Proof Let $m$ be the cardinality of $I$. If the $a_{i}$ 's are all in the interior of $D(z, y, s)$, then there exists $\tilde{s} \in] 0, s]$ such that $\left\{a_{1}, \ldots a_{m}\right\} \subseteq D(z, y, \tilde{s})$, contradicting the minimality of $s$. Hence, at least one of the $a_{i}$ 's belongs to the boundary of $D(z, y, s)$. Suppose for instance that

$$
\begin{aligned}
a_{1} & \in \operatorname{bd}[D(z, y, s)], \\
\left\{a_{2}, \ldots, a_{m}\right\} & \subseteq \operatorname{int}[D(z, y, s)] .
\end{aligned}
$$

We must arrive to a contradiction. If one sets

$$
c_{1}:=\frac{a_{1}-z}{\left\|\left(a_{1}-z\right)\right\|}, \quad y_{t}:=\frac{y-t\left(c_{1}-y\right)}{\left\|y-t\left(c_{1}-y\right)\right\|}, \quad s_{t}:=\left\langle c_{1}, y_{t}\right\rangle,
$$

then one can prove that $\left\{a_{1}, \ldots a_{m}\right\} \subseteq D\left(z, y_{t}, s_{t}\right)$ for all $t>0$ small enough. On the other hand, the right-derivative at 0 of the function $t \mapsto s_{t}$ is given by

$$
\left(\frac{d s_{t}}{d t}\right)_{t=0^{+}}=\left\langle c_{1}, y\right\rangle-1<0 .
$$

Hence, $s_{t}<s$ for all $t$ near 0 , contradicting again the minimality of $s$. 
We end this paper by stating a min-max formulation of the penumbra problem. Following the same pattern as in Sect. 2.3, we start by writing

$$
s_{\diamond}=\inf _{z \in Z} \Psi(z),
$$

where $\Psi(z)$ denotes the optimal value of the lower dimensional problem

$$
(\mathcal{Q})_{z}\left\{\begin{array}{l}
\text { minimize } s \\
(y, s) \in \mathbb{S}_{n} \times[0,1] \\
f_{i}(z, y, s) \geq 0 \text { for all } i \in I .
\end{array}\right.
$$

Unfortunately, the function $\Psi$ is not as simple to evaluate as $\Phi$. The following result corresponds to the penumbra counterpart of Theorem 2.12. The notation

$$
\Delta_{m}:=\left\{\lambda \in \mathbb{R}^{m}: \lambda_{1} \geq 0, \ldots, \lambda_{m} \geq 0, \sum_{i=1}^{m} \lambda_{i} \leq 1\right\}
$$

refers to the full dimensional simplex in $\mathbb{R}^{m}$.

Theorem 4.3 Let $m$ be the cardinality of $I$. Then $\Psi: A^{c} \rightarrow \mathbb{R}$ is continuous and admits the representation

$$
\Psi(z)=\min _{\|y\|=1} \sigma(z, y),
$$

where $\sigma(z, \cdot)$ is the nonnegative sublinear function given by

$$
\sigma(z, y):=\max \left\{0,\left\langle\mathbf{c}_{1}(z), y\right\rangle, \ldots,\left\langle\mathbf{c}_{m}(z), y\right\rangle\right\} .
$$

In particular, if $Z$ is included in $\operatorname{int}(P)$ and does not intersect $A$, then the optimal value of (4) admits the min-max formulation

$$
s_{\diamond}=\min _{\substack{z \in Z \\\|y\|=1}} \max _{\lambda \in \Delta_{m}}\left\langle\sum_{i=1}^{m} \lambda_{i} \mathbf{c}_{i}(z), y\right\rangle .
$$

Proof Since the variable $s$ is to be minimized in (52), one can drop the constraint $s \leq 1$. On the other hand, the last constraint in (52) can we written in the form $\left\langle\mathbf{c}_{i}(z), y\right\rangle \leq s$ for all $i \in\{1, \ldots, m\}$. Keeping in mind that $s$ must also satisfy the contraint $s \geq 0$, one deduces that $\Psi(z)$ is equal to the optimal value of the minimization problem

$$
\left\{\begin{array}{l}
\operatorname{minimize} s \\
\|y\|=1 \\
\max \left\{0,\left\langle\mathbf{c}_{1}(z), y\right\rangle, \ldots,\left\langle\mathbf{c}_{m}(z), y\right\rangle\right\} \leq s .
\end{array}\right.
$$

This leads to the characterization (53) and proves the continuity of $\Psi$ on $A^{c}$. The second part of the theorem is obtained by combining (51) and (53).

\section{References}

1. Ahn, H.K., Bae, S.W., Cheong, O., Gudmundsson, J.: Aperture-angle and Hausdorff approximation of convex figures. Discret. Comput. Geom. 40, 414-429 (2008)

2. Astorino, A., Fuduli, A.: Nonsmooth optimization techniques for semisupervised classification. IEEE Trans. Pattern Anal. Mach. Intell. 29, 2135-2142 (2007)

3. Astorino, A., Fuduli, A., Gaudioso, M.: DC models for spherical separation. J. Glob. Optim. 48, 657-669 (2010) 
4. Astorino, A., Fuduli, A., Gaudioso, M.: Margin maximization in spherical separation. Comput. Optim. Appl. 53(2), 301-322 (2012)

5. Astorino, A., Gaudioso, M., Seeger, A.: Conic separation of finite sets. I. The homogeneous case. J. Convex Anal. (2012, to appear)

6. Aubin, J.-P., Frankowska, H.: Set-Valued Analysis. Birkhäuser, Boston (1990)

7. Bose, P., Hurtado-Diaz, F., Omana-Pulido, E., Snoeyink, J., Toussaint, G.T.: Some aperture-angle optimization problems. Algorithmica 33, 411-435 (2002)

8. Clarke, F.H.: Generalized gradients and applications. Trans. Am. Math. Soc. 205, 247-262 (1975)

9. Coban, C.K.: Model-based synthesis of sensor location. In: Proceedings of IEEE International Conference on Robotics and Automation, vol. 2, pp. 900-905 (1988)

10. Danskin, J.M.: The Theory of Max-Min and Its Application to Weapons Allocation Problems. Springer, New York (1967)

11. Dörrie, H.: Hundred Great Problems of Elementary Mathematics: Their History and Solution. Translated by D. Antin, Dover Publications, New York (1965)

12. Dunkl, C.F., Williams, K.S.: A simple norm inequality. Am. Math. Mon. 71, 53-54 (1964)

13. Frangioni, A.: Solving semidefinite quadratic problems within nonsmooth optimization algorithms. Comput. Oper. Res. 23, 1099-1118 (1996)

14. Fuduli, A., Gaudioso, M., Giallombardo, G.: Minimizing nonconvex nonsmooth functions via cutting planes and proximity control. SIAM J. Optim. 14, 743-756 (2004)

15. Gourion, D., Seeger, A.: Solidity indices for convex cones. Positivity 16, 685-705 (2012)

16. Henrion, R., Seeger, A.: On properties of different notions of centers for convex cones. Set-Valued Var. Anal. 18, 205-231 (2010)

17. Henrion, R., Seeger, A.: Inradius and circumradius of various convex cones arising in applications. SetValued Var. Anal. 18, 483-511 (2010)

18. Henrion, R., Seeger, A.: Condition number and eccentricity of a closed convex cone. Math. Scand. 109, 285-308 (2011)

19. Iusem, A., Seeger, A.: Normality and modulability indices. I. Convex cones in normed spaces. J. Math. Anal. Appl. 338, 365-391 (2008)

20. Kiwiel, K.C.: A method for solving certain quadratic programming problems arising in nonsmooth optimization. IMA J. Numer. Anal. 6, 137-152 (1986)

21. Moreno, J.P., Seeger, A.: External analysis of boundary points of convex sets: supporting cones and drops. J. Convex Anal. 16, 939-957 (2009)

22. Omana-Pulido, E., Toussaint, G.T.: Aperture-angle optimization problems in three dimensions. J. Math. Model. Algorithms 1, 301-329 (2002)

23. Wolfe, P.: Finding the nearest point in a polytope. Math. Program. 11, 128-149 (1976) 